\title{
Purification and Partial Characteristics of Polysaccharides from Rosa Laevigata Michx Fruits
}

\author{
Xuejiao Zhang, Chenzhong Jin, Yihong Hu,Yunyun Zhou, Shuanghui Wang \\ Hunan University of Humanities, Science and Technology \\ Dixing road487, Loudi, China \\ 380711974@qq.com; 532479626@qq.com; 33379121@qq.com; 67547204@qq.com; 125904806@qq.com
}

\section{Extended Abstract}

Extraction optimization, purification and partial characteristics of polysaccharides from the traditional Chinese herb Rosae Laevigatae Michx were investigated. R. Leavigata belongs to the Rosa genus and Rosaceae family, and its fruits are widely used as health food in China, Japan and the other Asian countries [1]. In this study, the response surface methodology was used to optimize the extraction conditions of water-soluble polysaccharides from $R$. Laevigatae fruits. The central composite design was used to optimize the extraction processing parameters. The optimum extraction conditions were extraction temperature $95^{\circ} \mathrm{C}$, extraction time $2.5 \mathrm{~h}$, water to raw material ratio $22: 1$, and extraction frequency 3 . Under the optimum conditions, the experimental yield was $9.55 \pm 0.1 \%$, which is in good agreement with the predicted yield. Six major fractions (RLP-I-1, RLP-I-2, RLP-I-3, RLP-II-1, RLP-II-2 and RLP-II-3) were successfully obtained by purifying the crude polysaccharides extracted from the fruits by using diethylaminoethanol-Sepharose column chromatography and Sepharose CL-4B column chromatography respectively. The molecular weights of major fractions were determined by the high performance gel permeation chromatography with a differential refractive index detector (Waters2410, USA) and a G5000 PWxl column $(7.8 \mathrm{~mm} \times 300 \mathrm{~mm}$, TOSOH, Japan) connected in series with a G3000 PWxl column $(7.8 \mathrm{~mm} \times 300 \mathrm{~mm}$, TOSOH, Japan). The average molecular weight of the RLP-I-1, RLP-I-2, RLP-I-3, RLP-II-1, RLP-II-2, RLP-II-3 were estimated to be $8930 \mathrm{Da}, 9498 \mathrm{Da}, 9662 \mathrm{Da}, 7673 \mathrm{Da}, 8558 \mathrm{Da}$ and $8323 \mathrm{Da}$, respectively. The fourier transform infrared spectra of the six polysaccharides were identical, and the broad stretching intense characteristic peak was shown at $3400 \mathrm{~cm}^{-}$ ${ }^{1}$ for the -OH group, whereas a weak C-H stretching band was observed from 2935-2939 $\mathrm{cm}^{-1}$. The peaks at $1616 \mathrm{~cm}^{-1}$ were from the bending vibration absorption of $-\mathrm{OH}$ group. Absorption peaks ranged from $1460-1200 \mathrm{~cm}^{-1}$ were the variable angle vibrations of $\mathrm{C}-\mathrm{H}$. The peaks from $1285 \mathrm{~cm}^{-1}$ to $1020 \mathrm{~cm}^{-1}$ corresponded to $\mathrm{C}-\mathrm{O}$ stretching vibrations. The absorption band round $880 \mathrm{~cm}^{-1}$ was due to the $\alpha$-type glycosidic bond. Peaks ranged from $3600-3200 \mathrm{~cm}^{-1}$ and $1655-1615 \mathrm{~cm}^{-1}$ revealed that these compounds were carbohydrates. Moreover, no peaks were detected near $1715 \mathrm{~cm}^{-1}$ suggesting that the fructan had no uronic acid [2]. In the present study, the anti-oxidative activities of the six polysaccharides toward super-oxide anion, hydroxyl, and 1, 1-diphenyl-2-picrylhydrazyl (DPPH) radicals in vitro were evaluated in terms of scavenging rate. The assays suggested that RLP-II-1, RLP-II-2 and RLP-II-3 presented higher scavenging activity toward superoxide anions, hydroxyl and DPPH radicals. The results indicated that the response surface methodology was an effective method for the extraction of polysaccharides from the $R$. Laevigatae fruits, and the polysaccharides could be explored as a potential antioxidant agent for use in functional foods.

\section{References}

[1] C. Yu, X. Dai, Q. Chen, J. Zai, L. Deng, Y. Liu, H. Ying, "Hypolipidemic and antioxidant activities of polysaccharides from Rosae Laevigatae Fructus in rats," J. Carbohydrate Polymers, vol. 94, no. 1, pp. 56-62, 2013.

[2] C. Nie, P. Zhu, S. Ma, M. Wang, Y. Hu, "Purification, characterization and immunomodulatory activity of polysaccharides from stem lettuce," J. Carbohydrate Polymers, vol. 188, pp. 236-242, 2018. 